
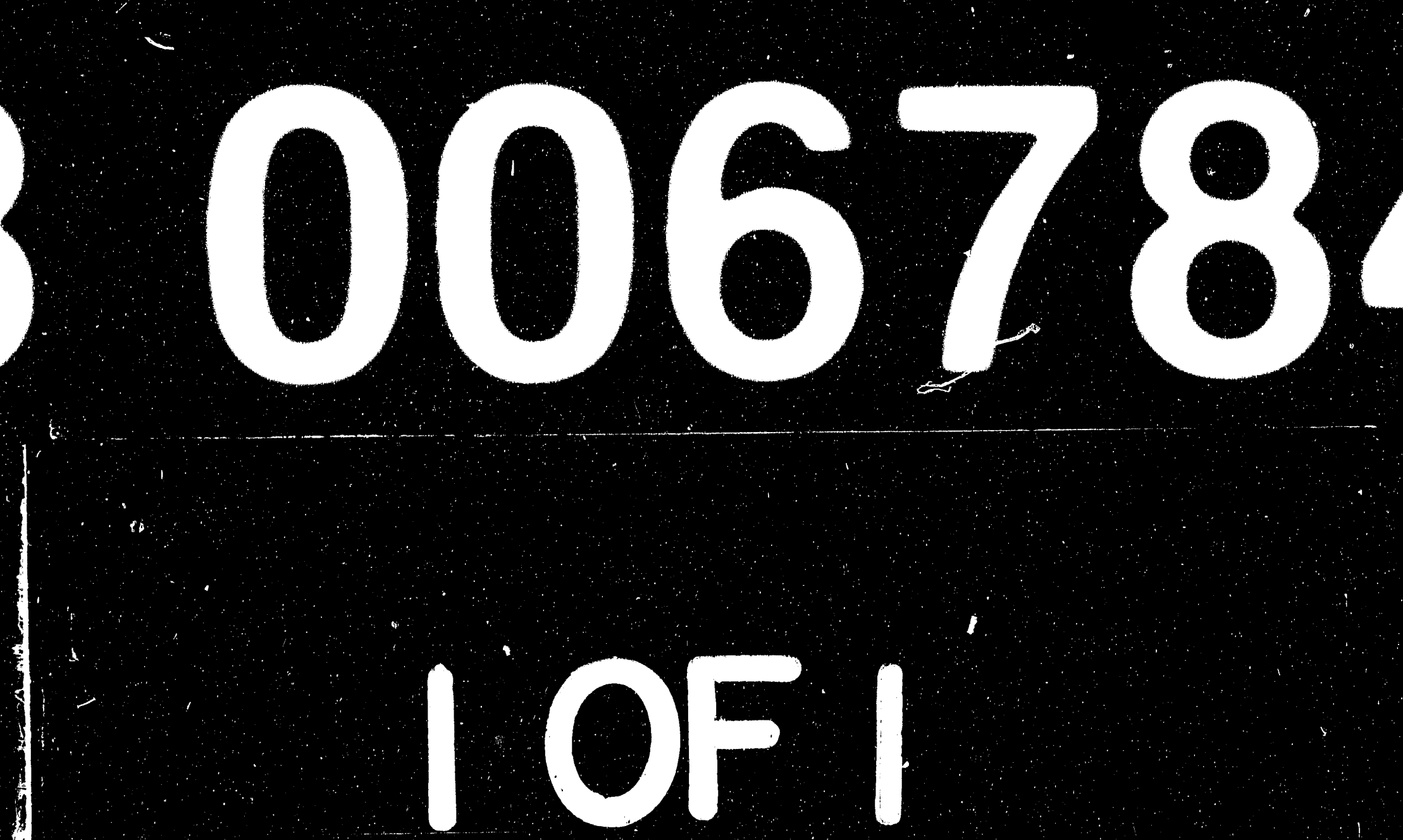

1.

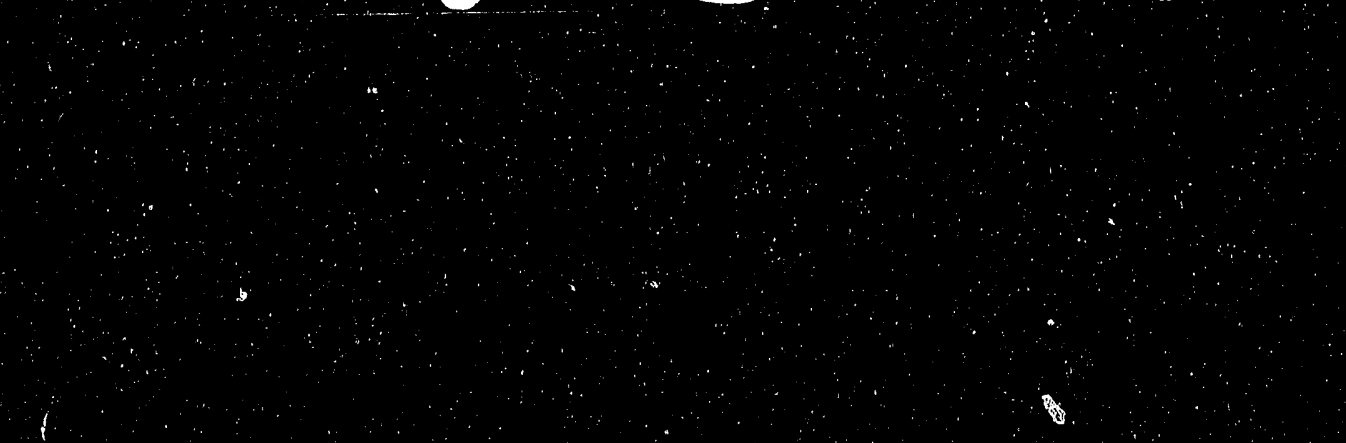

11

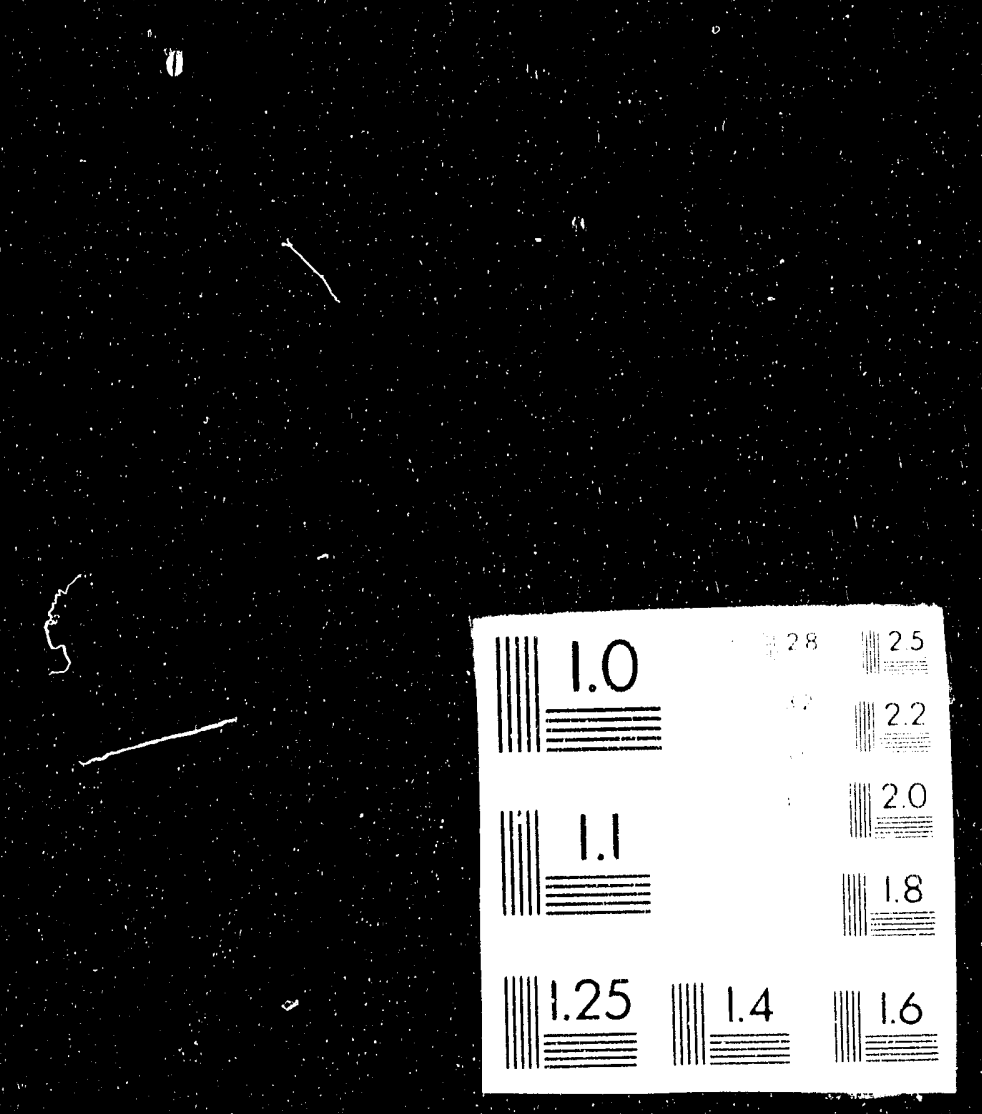


DOE/ER/13905--5

DE93 006784

\title{
PHOTOINDUCED ELECTRON TRANSFER IN ORDERED POLYMERS
}

PROGRESS REPORT

\author{
January 1, 1992 - December 31, 1992
}

Guilford Jones, II

Department of Chemistry, Boston University

Boston, Massachusetts 02215

January 1993

Prepared for the

U. S. Department of Energy

under contract no. DE-FG02-88ER 13905 


\section{PHOTOINDUCED ELECTRON TRANSFER IN ORDERED POLYMERS}

Long range photoinduced electron transfer between electron donor and acceptor groups is of considerable current interest in terms of strategies for aritificial photosynthesis and studies regarding the redox properties of proteins. As part of an extensive investigation of long range electron transfer involving biopolymers, we have carried out this year investigations of the assembly of electron transfer agents in various systems, including model short peptides, peptide electrolytes, and peptide template catalysts. The principal interest in these systems has to do with the control over spacial dimensions (distances of separation between electron donors and acceptors) and the operation of various linkages that utilize organic functional groups (some with unusual geometries) as spacers or "conduits" for long-range electron transfer. Also of interest are the well ordered secondary structures (e.g., the alpha-helices) adopted by peptide polymers, and the capabilities for synthetic modification of peptide side chains and end groups with chromophores or electroactive substituents.

The present report gives a brief account of the following elements of work related to photochemical electron transfer themes: (1) the synthesis and photochemical characterization of chromophore-bound peptides and amino acid model compounds based on the amino acids, tryptophan and the spacer residue, alanine (Ala); (2) the study of binding of cationic organic dyes (e.g., styryl-7) to a peptide electrolyte, for which cooperative dye loading and helix formation is important; (3) the completion of work on a new series of acridinium chromophores that have "rod-like" arrangements of linked aryl rings for assembly of electron donor-acceptor systems that exhibit long lived charge separation; and (4) use of the modified form of the peptide, poly-L-histidine ( $\mathrm{PLH})$, as a template for sulfide oxidation (development of a peptide "catalytic triad").

Peptides and amino acids modified with pyrene chromophores. Modified peptides consisting of the naturally occurring amino acids, L-tryptophan (Trp), and L-alanine (Ala) (Scheme 1) have been prepared. These amino acid derivatives contain a chromophoric group, pyrene sulfonamide (Pyr), covalently bound at the $\mathrm{N}$ terminus, and an electron donor moiety, dimethylphenylenediamine (DMPD) located at the C-terminus (and including other model variations). Photochemical electron transfer involving the bound 
chromophore and the peptide chains have been carried out through a collaboration with the group of Dr. Michael Wasielewski at Argonne National Laboratory. Picosecond time-resolved absorption measurements have revealed in preliminary work that the forward rate constants for electron transfer between excited pyrene and Trp or DMPD groups occur in the $10 \mathrm{psec}$ time regime. Significantly, the back electron transfer occurs in the nsec time domain. The latter depends dramatically on the substitution pattern (distance between terminal groups) and provides an example of "inverted region" behavior for a modified biopolymer system. An important problem that is to be resolved has to do with the role of an aromatic side chain in a Trp moiety in controlling the rate of long distance electron transfer between terminal moieties, Pyr and DMPD. The systematic placement of Trp moieties in tripeptides (e.g., Pyr-Ala-Trp-AlaDMPD) provides a unique look at this factor that is of potential importance in the control of rates of charge recombination (separation) in proteins.

Self-assembled biopolymer - dye complexes: induction of a peptide helix for chromophore-bound polyglutamate. Our studies of polyelectrolytes and their role in the binding of charged organic dyes in water continues with the investigation of poly-(L-glutamic acid) (PGlu or PLGL) which readily binds dyes of the cyanine or merocyanine type. The most novel feature that we have recently discovered for these systems is the tendency of the dye to form dimer aggregates when the chromophore is electrostatically bound, and, remarkably, the tendency of the charged peptide to fold into a helical conformation on interaction with dye counterions (at relatively high loading). Of principal importance in these systems is the ability of peptide-dye complexes to self-assemble into different biopolymer structural motifs that exhibit long range (i.e., onedimensional, helical) order and arrays of chromophores "stacked" along biopolymer chains. These systems hold particular promise for demonstration of entrainment of charge over nanometer dimensions.

The complex of PIC with the conventional polymer, poly(acrylic acid) (PAA), in water at a proper $\mathrm{pH}$ for charging of the polyelectrolyte, results in dramatic alteration of the absorption spectrum (development of a relatively sharp, intense "J-band"), as shown in Figure 1. This feature is the result of the formation, at high dye loading on the polymer, of a "brick-work" motif of stacked chromophores known as J-aggregates. Alternatively, a merocyanine dye such as styryl-7 (S7) adopts a different geometrical array on 
polymer binding whose signature is the blue-shift of absorption, the result of "H-type" aggregation associated with a "card-pack" arrangement of chromophores (dye dimers predominately). The spectra for S7 bound as counter-ion to the peptide, poly-L-(glutamic acid) (P-Glu) are shown in Figure 2, as a function of $\mathrm{pH}$ for water solutions and a selected concentration ratio of peptide residues to dye $(P / D=2)$.

A most novel feature that accompanies the formation of electrostatically bound dye aggregates is the tendency of the charged peptide, polyglutamate, to fold into a helical conformation on interaction with dye counterions (at relatively high loading). Along with the absorption characteristics of styryl-7 (S7) on binding to PGlu, shown below in Figures 3-5 are (1) the induction of circular dichroism ( $C D$, optical activity) in dye dimers on binding to peptide in two conformational forms, whose relative importance depends on $\mathrm{pH}$ in a sensitive way, (2) the simultaneous onset of induced CD (ICD), in the visible, associated with bound dye dimers, and the adoption of the helix conformation (negative dichroic bands at 208 and $220 \mathrm{~nm}$ ) for the PLGL peptide as a function of $\mathrm{pH}$, and (3) the "saturation" (adoption of complete helix structure) on loading of sufficient dye counter-ions. Of principal importance in these systems is the ability of peptide-dye complexes to self-assemble into different biopolymer structural motifs that exhibit long range (i.e., one-dimensional, helical) order, and arrays of chromophores "stacked" along biopolymer chains. These systems hold particular promise for demonstration of entrainment of excitation energy or charge over nanometer dimensions. A molecular model (CHARMm calculation) that illustrates the binding of $S 7$ dimer to PGlu is shown in Figure 6. The dramatic change in the sign of induced $C D$ with medium conditions is presumably a function of how local transition moments associated with dye aggregate are aligned lone arrangement in Figure 6) relative to the direction of dye stacking on the PGlu polyelectrolyte that is changing its shape (conformation) as a function of its electrostatic charge (medium $\mathrm{pH}$ ).

"Twisted" biaryl donor-acceptor structures based on the acridine chromophore. Parallel studies of primary photochemical electron transfer events include the development of new sensitizers that are capable of "internal" charge displacement. The new photosensitizers are associated with extremely rapid and efficient separation of charge and the formation of reactive intermediates with a wide range or lifetimes, suitable for the further entrainment 
of charge within a matrix or the promotion of secondary redox reactions. The synthesis of a new series of acridinium chromophores that have "rod-like" arrangements of linked aryl rings has been recently completed. These structures are "tuned" to allow rather precise control of the rate of charge shift and charge recombination processes. The new structures, shown in Figure 7, exhibit the important feature of linkages that have, as their principal geometric variable, rotation about single bonds that link aromatic rings. These rotations are hindered to an extent that is subtle but predictable, therefore providing a control on the degree of interaction (orbital overlap) between electron donor (aromatic or phenolic) and electron acceptor (acridinium) segments. The expectation is that a systematic adjustment of the length and torsional flexibility of these linkages will provide a means for demonstrating very long lived charge separation, even over relatively short distances (0.5-1.0 nm) and the testing of limits to electronic coupling necessary for efficient forward and back electron transfer. Furthermore, these chromophores are designed so that as charged species they can bind to electrolyte biopolymers (e.g., PGlu) or be covalently bound to peptides having other electroactive groups.

The fluorescence for the donor substituted acridiniums is illustrated in Figure 8 where a comparison is made between the naphthyl substitution with a substituent (phenyl) having poor electron donating properties. For the latter structure the emission spectrum is essentially that of unsubstituted acridinium $(510 \mathrm{~nm})$. We therefore assign to the acridiniums that display a red-shifted emission a low lying charge shift (CS) transition. This emission occurs on excitation of the lightly perturbed local (LE) absorption (Figure 7). Notably, the CS emission band that appears at 540-630 $\mathrm{nm}$ is only moderately dependent on solvent polarity, over a range of 80 in dielectric constant (a solvent series ranging from chloroform --> water may be readily used!). The yield is more strongly dependent on substitution pattern with quantum yields that vary from a few tenths (for naphthyl substitution) to $<10^{-3}$ for dyes with the better donor groups (phenol). Picosecond absorption measurements have been carried out at both the Argonne National Laboratory (Wasielewski group) and the Center for Fast Kinetics at the University of Texas at Austin. Radical-pair species have been observed (Figure 9) that display lifetimes of a few nanosecounds, corresponding to the flourescence decays for the CS states. 
The spin polarization of triplet species has been also determined through a flash ESR study of the acridinium dyes in which the pathway CS (singlet radical pair) $\rightarrow$ LE (local acridinium triplet) provides a special signature. This work (paper in preparation) has been carried out with the collaboration of Professor Hans van Willigen of the University of Massachusetts, Boston. This series of structires and the wide variation in medium provide an important matrix with which mechanisms of electron transfer and solvation dynamics will be further elucidated.

Photocatalytic peptide: a redox catalytic triad for sulfide oxidation based on poly-L-histidine. PLH acts as a template for photocatalysis that is successful in converting a sulfide (a thianthrene derivative) to a sulfoxide. The peptide provides sites for covalent attachment of a sensitizer, for binding of a "substrate", and for the trapping of photogenerated sulfide radical cations. The latter step is central to the mechanism of two-electron sulfide oxidation. The mechanism of this photocatalysis is depicted in Schemes 2 and 3. In this work (publication below) laser photolysis experiments permitted the observation of initial electron transfer and radical ion trapping reactions that occur in the peptide domain. 


\section{RECENT PUBLICATIONS}

1. G. Jones, II and B. Huang, "Photoactive Peptides: Development of a Redox Catalytic Triad for Sulfide Oxidation," J. Phys. Chem., 1992, 96, 9603.

2. G. Jones, II and B. Huang, "Photoinduced Electron Transfer for High Potential Quinone Sensitizers and Thianthrenes. The Rate of Trapping of the Thianthrene Radical Cation by Water," Tetrahedron Lett., 1993, 34, 269.

3. G. Jones, II, B. Huang, and S. F. Griffin, "Electron Transfer Photochemistry of Thianthrene. Nucleophile Assisted Photooxidation to Sulfoxide," J. Org. Chem., in press.

5. G. Jones, II, and C. Oh, "Pseudoisocyanine in the Hydrophobic Microdomain of an Aqueous Polyelectrolyte: Fluorescence, Triplet and Electron Transfer Properties," J. Phys. Chem., submitted.

6. G. Jones, II, M. A. Farahat, and H. van Willigen, "Fourier Tranform ESR Study of the Mechanism of Intersystem Crossing for Electron Donor-Substituted Acridinium Salts. Spin Interactions in Short-link Electron Transfer Intermediates," in preparation. 

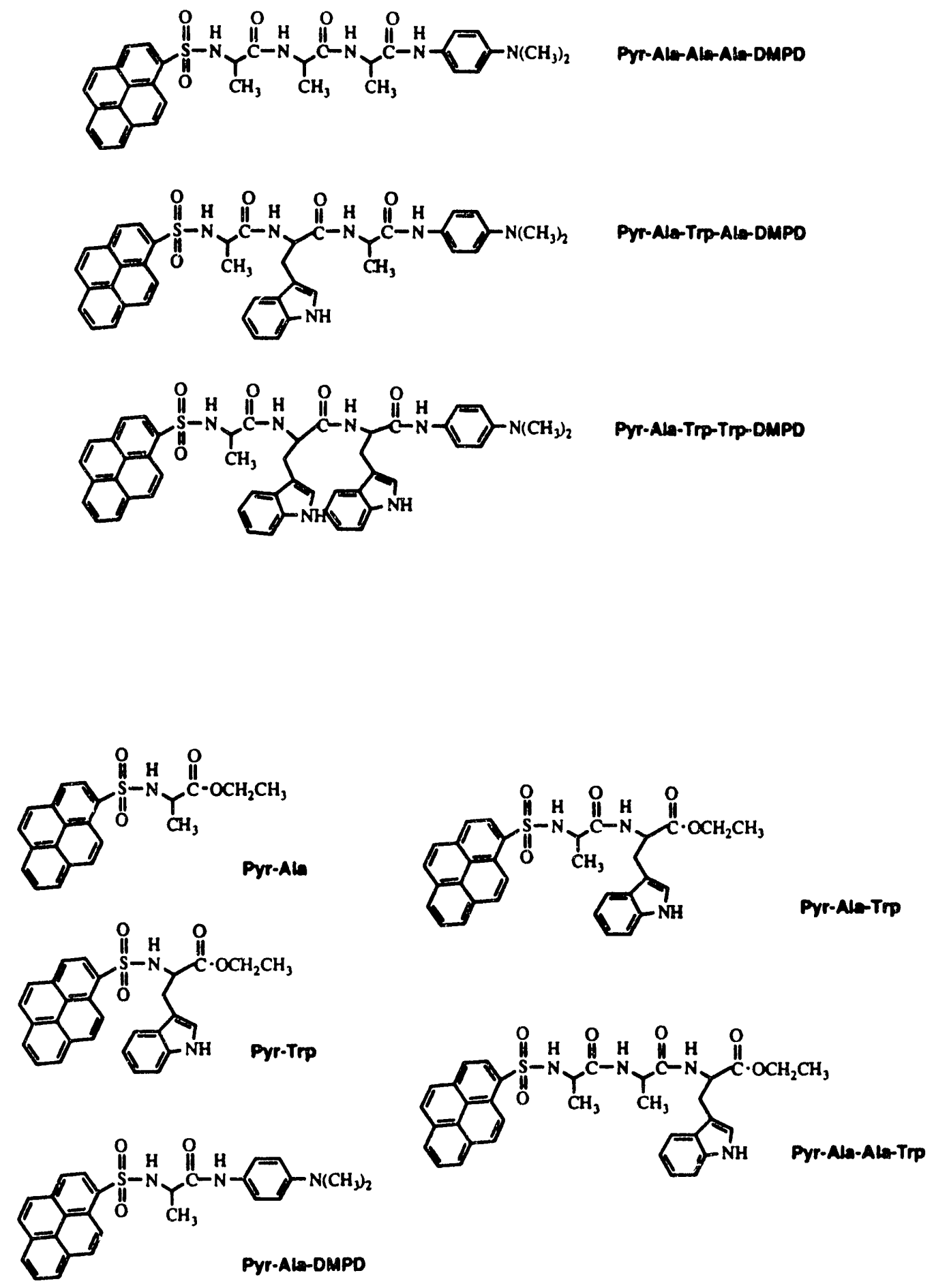

Pyr-Als-Tro 


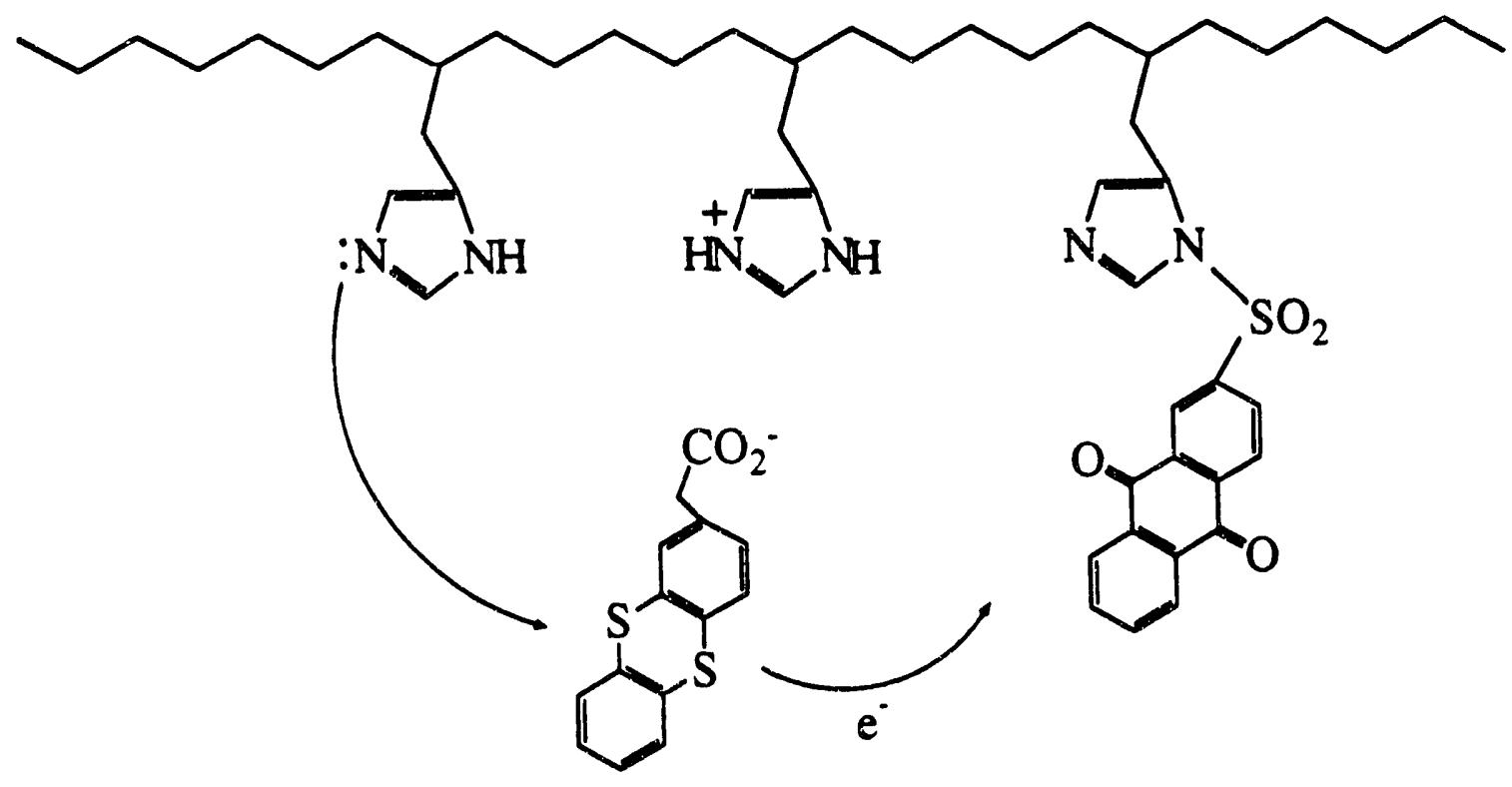

Free base side chain for nucleophilic trapping
Substrate sulfide quencher with electrostatic bond to template
Light harvesting sensitizer group and electron acceptor

Scheme 3

$$
\begin{aligned}
& -\mathrm{s}-\stackrel{-e^{\cdot}}{\longrightarrow}--_{\underline{s}}^{+}-\frac{\mathrm{HZ}}{\left(-\mathrm{H}^{+}\right)}-\stackrel{\mathrm{Z}}{-\underline{s}-}
\end{aligned}
$$

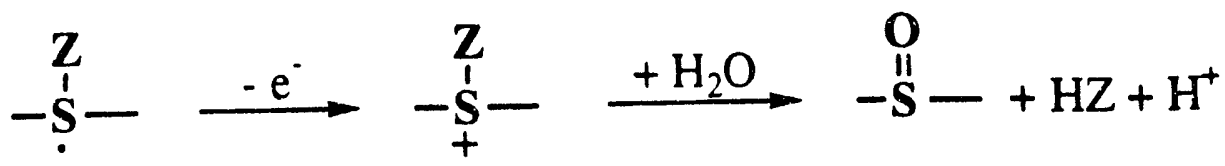




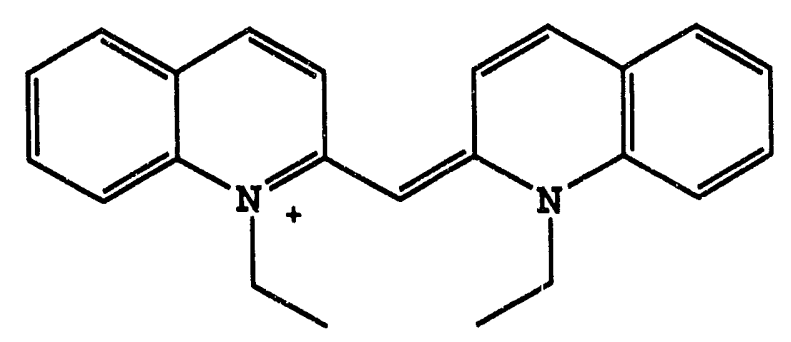

PIC

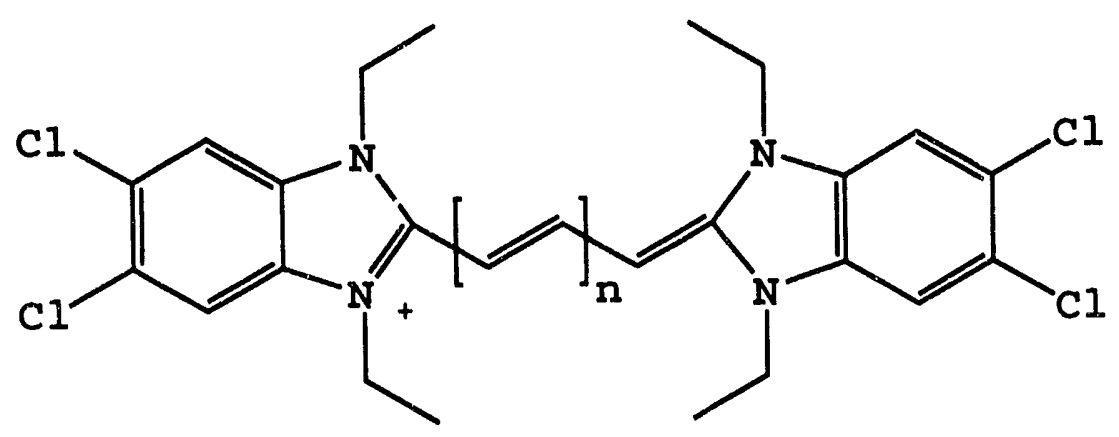

BIC

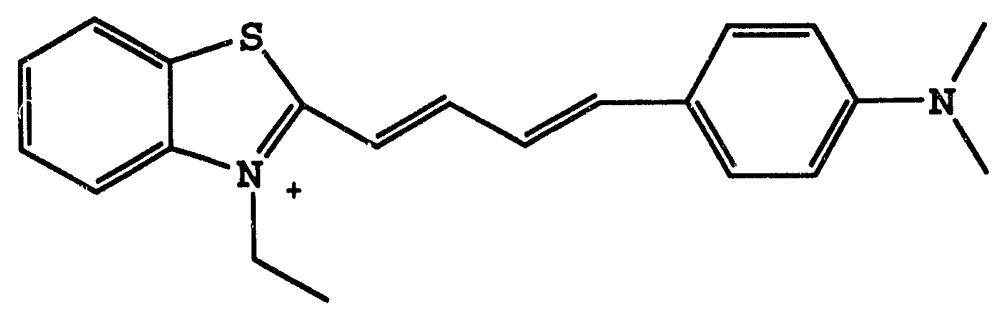

S7

$\overbrace{\mathrm{R}}^{\gamma_{\mathrm{nO}} \mathrm{H}}$

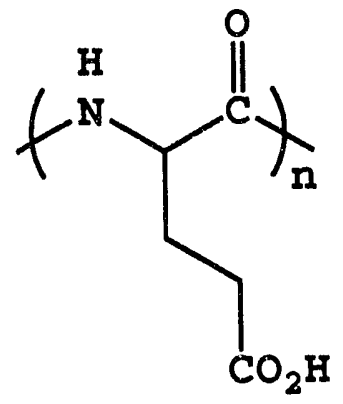

$\mathrm{R}=\mathrm{H}, \mathrm{PAA}$

p-Glu

${ }^{\prime}=\mathrm{Me}$, PMAA 


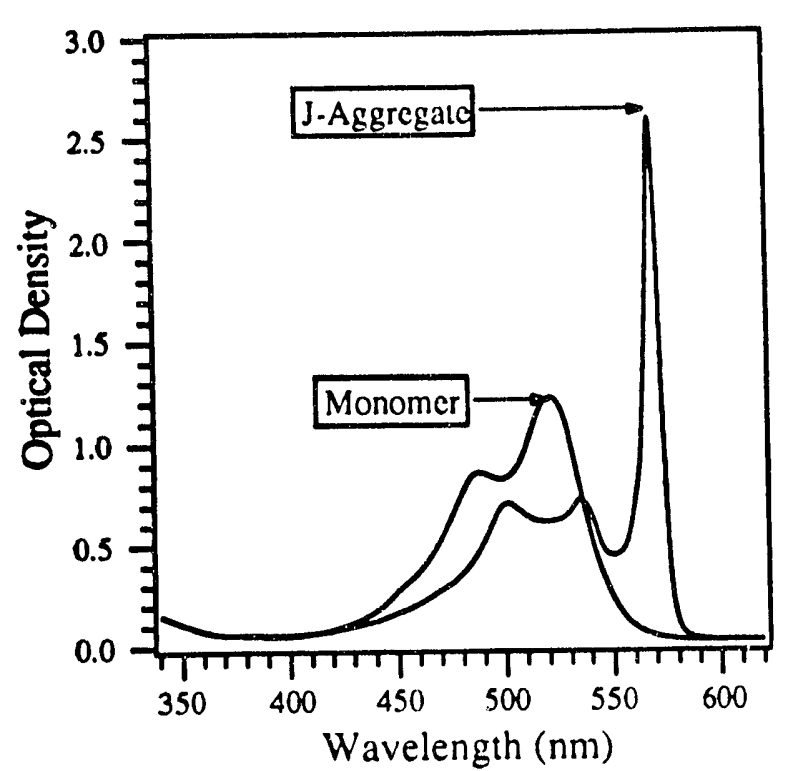

Figure 1 The absorption spectra for $30 \mu \mathrm{M}$ PIC in water, with (aggregate) and without PMAA polymer (P/D 6.0, pH 7.8).

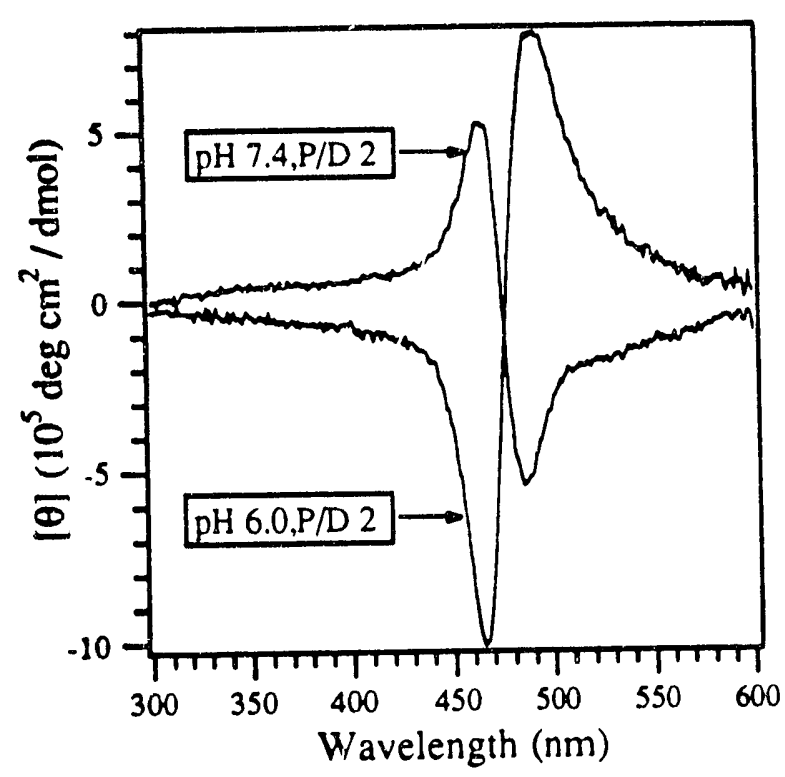

Figure 3 The ICD of S7-PLGL at P/D 2 $([S 7]=20 \mu \mathrm{M})$.

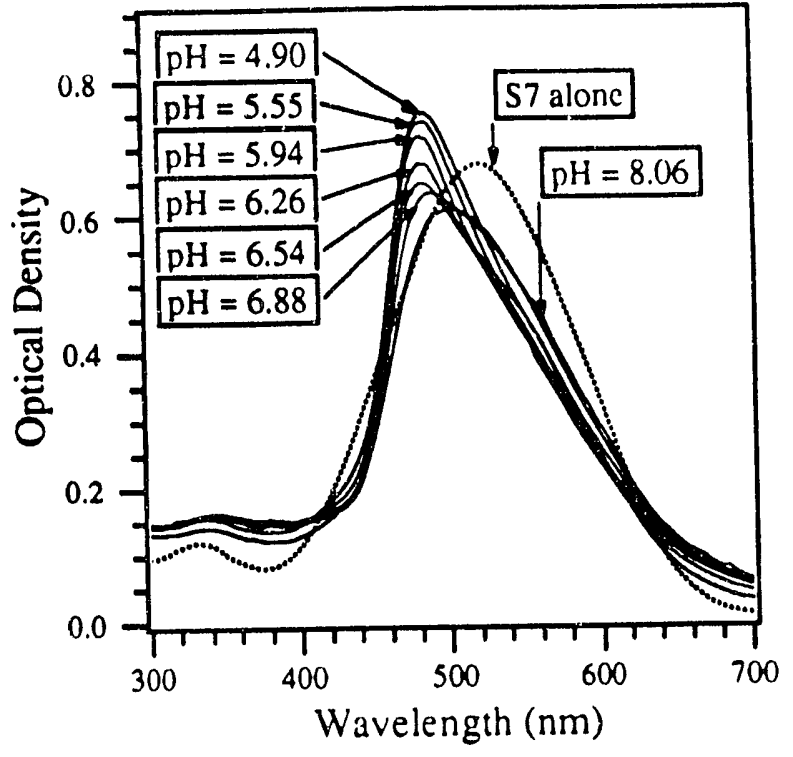

Figure 2 The absorption spectra for $20 \mu \mathrm{M}$ S7 without and with PLGL $(P / D=2)$ at various $\mathrm{pH}$.

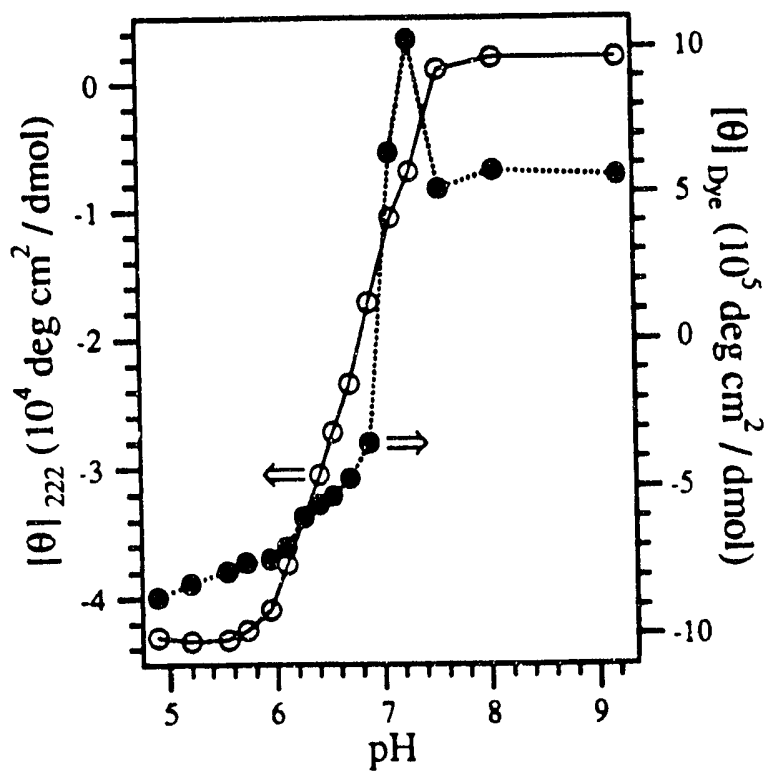

Figure 4 The pH dependence of $C D$ at 222 $\mathrm{nm}$ and ICD in the visible region for $\mathrm{S} 7$ PLGL $(P / D=2,[S 7]=20 \mu \mathrm{M})$. 


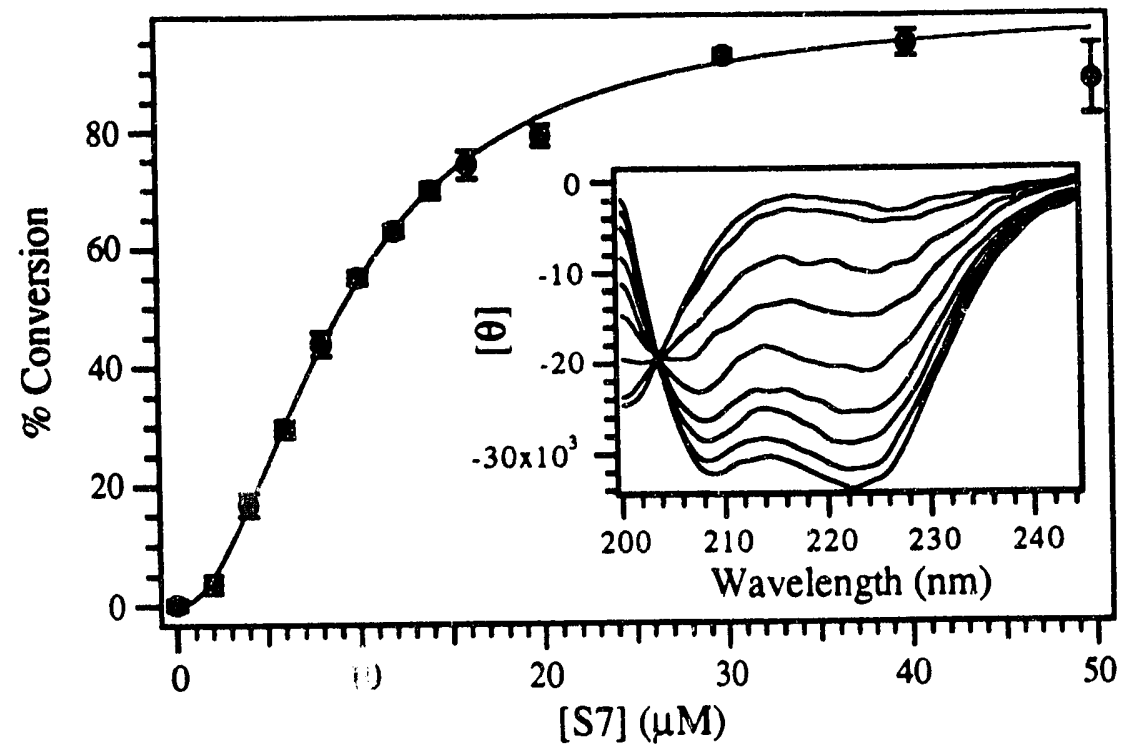

Figure 5 The P/D dependence of uv CD of S7-PLGL (pH 6.1, [PLGL] $=50 \mu \mathrm{M} ;[\mathrm{S} 7]=0,2,4$, $6,8,10,12,14,16 \mu \mathrm{M}$ from top in inset).

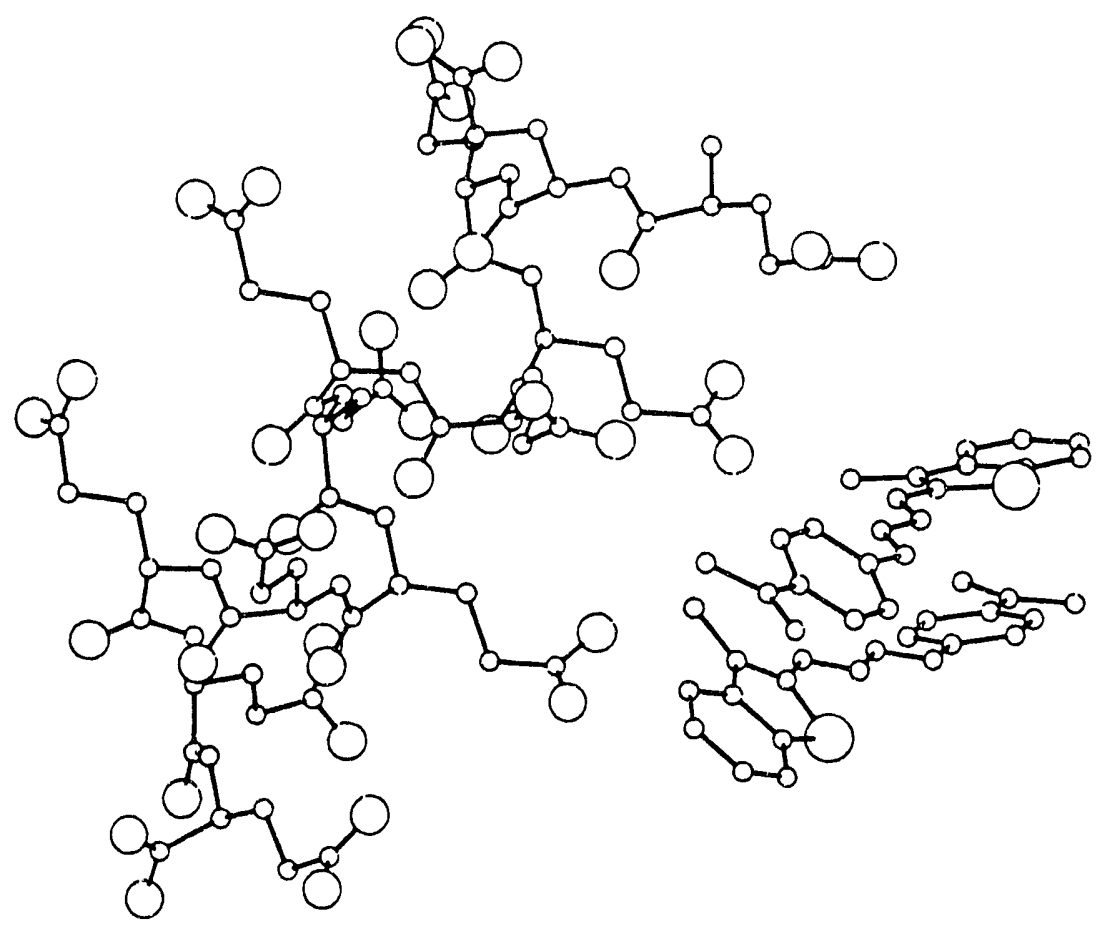

Figure 6 A computer model of the self-assembled PLGL/S7 dimer whose energy was minimized by CHARMm 


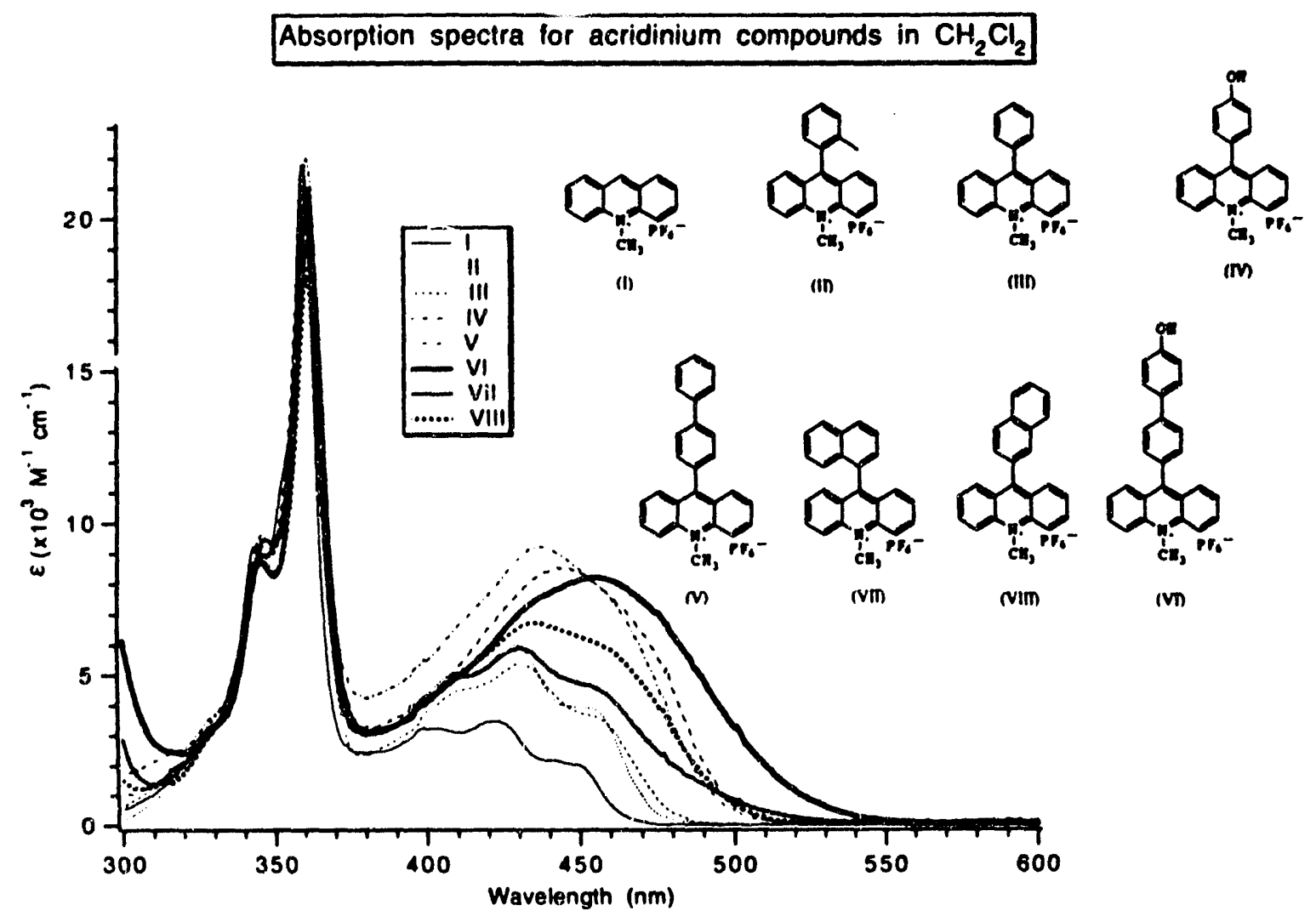

Figure 7

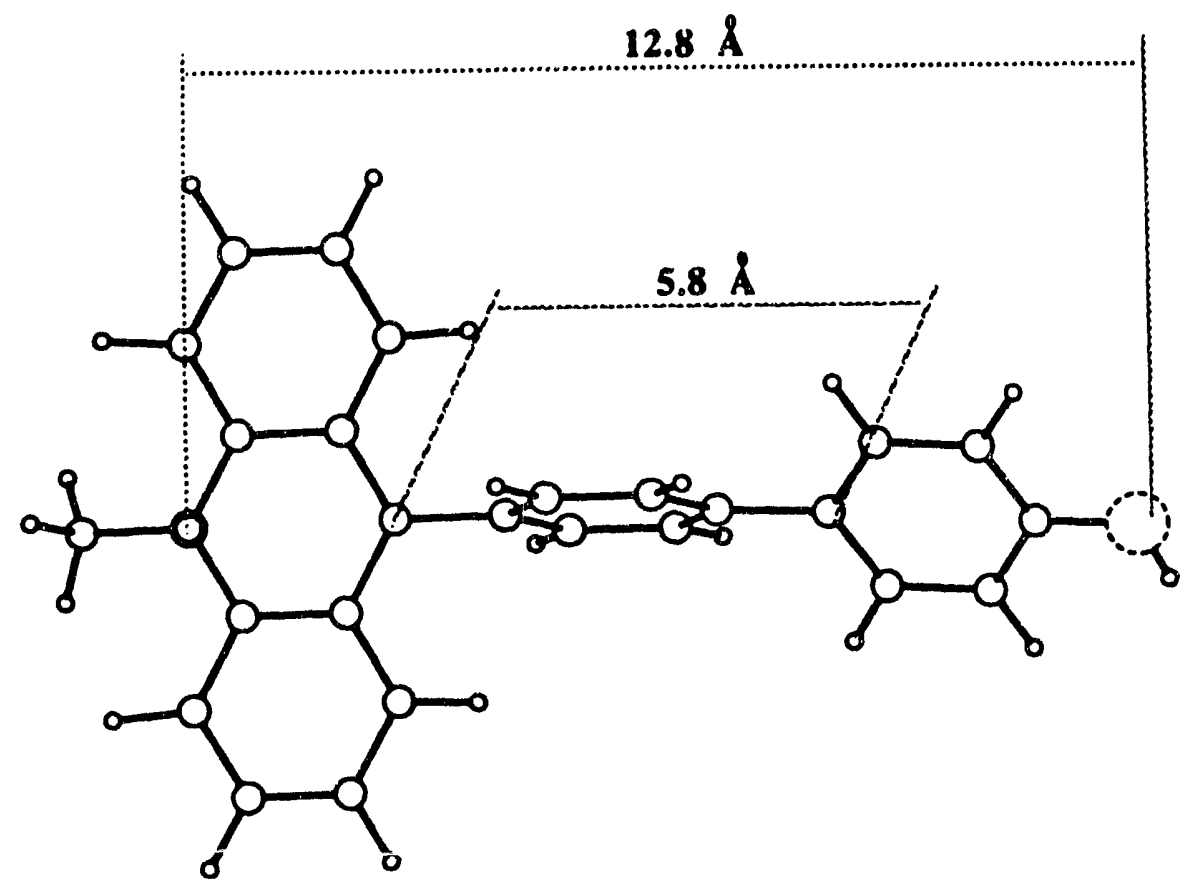




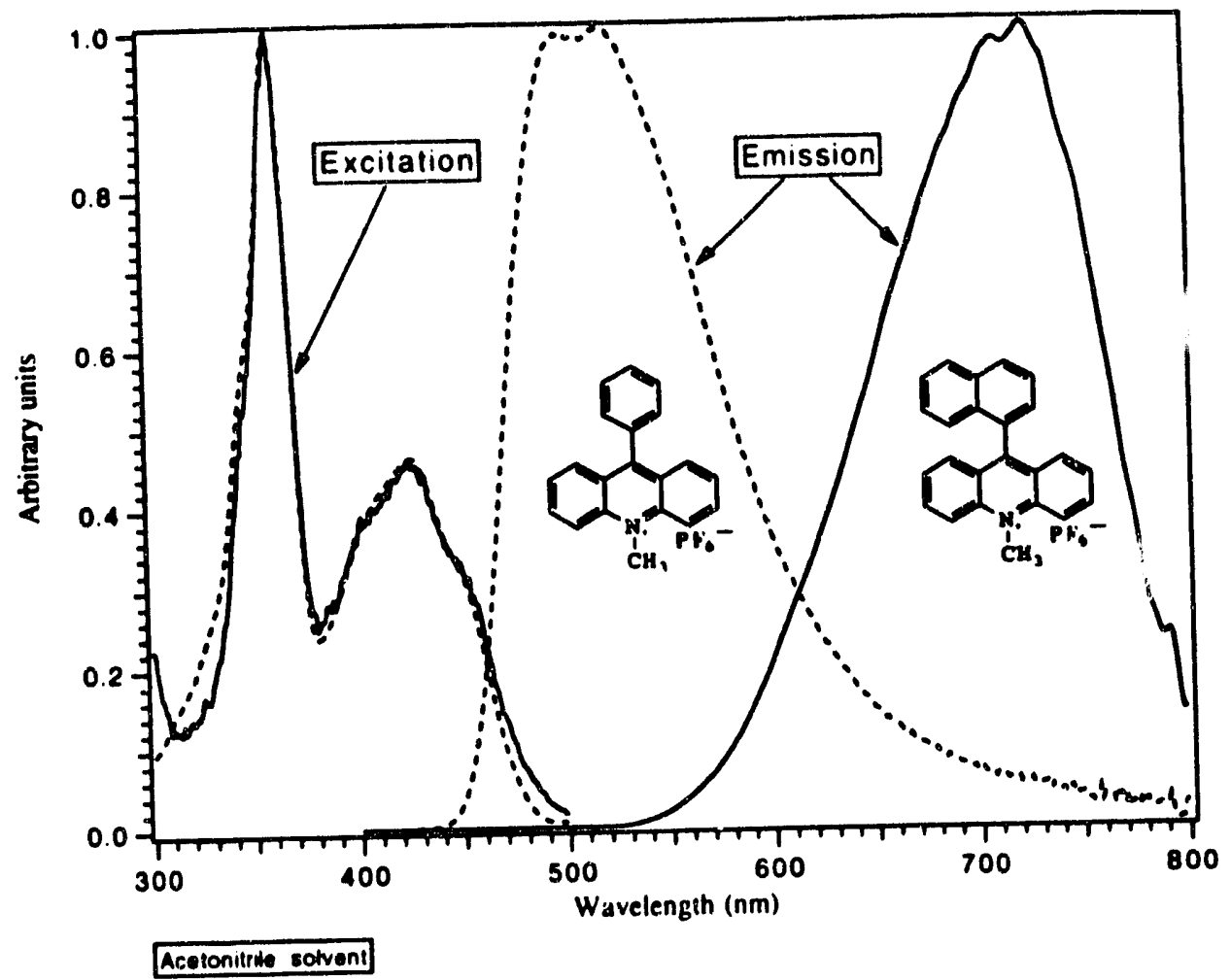

Figure 8

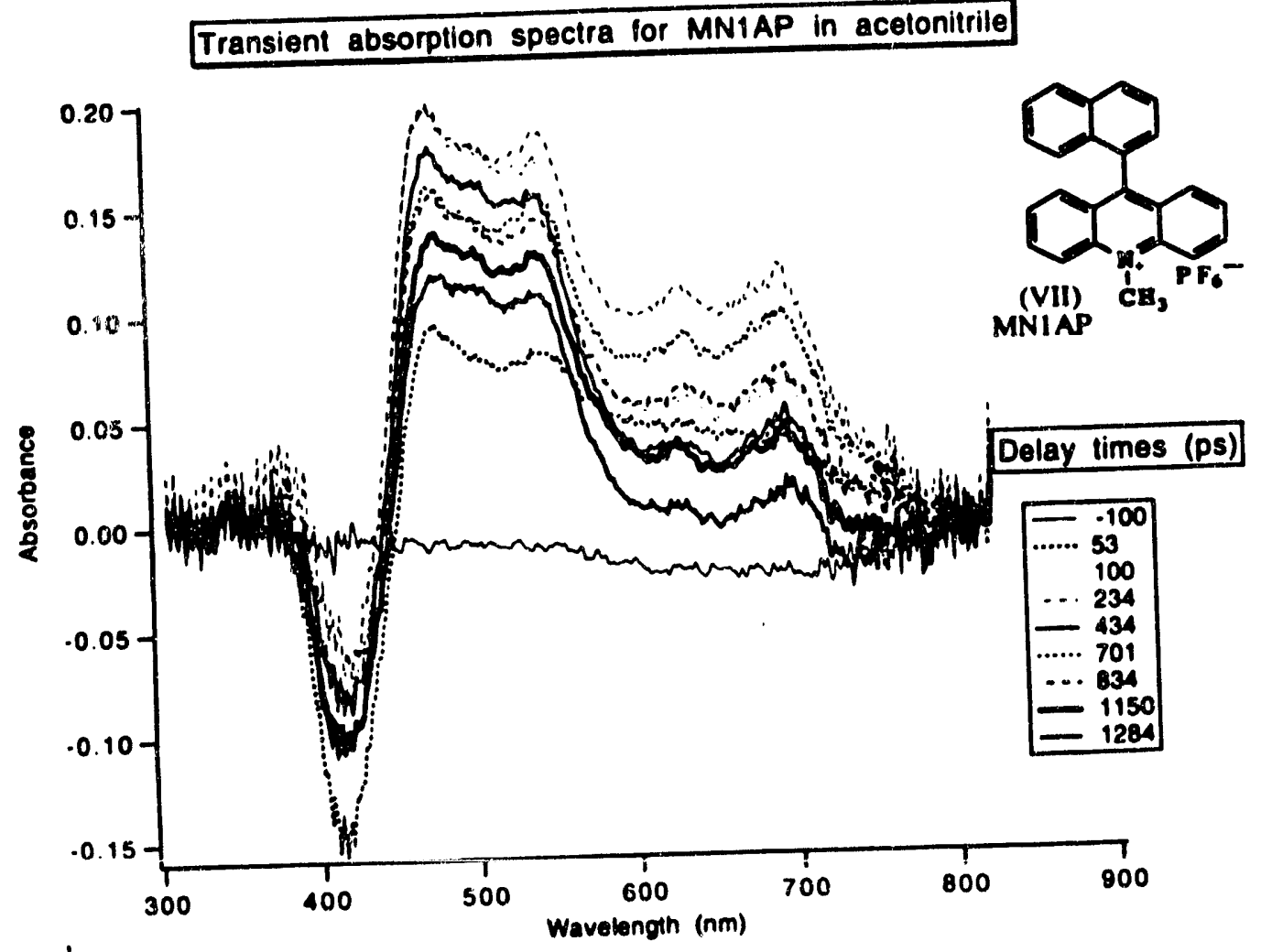

Figure 9 

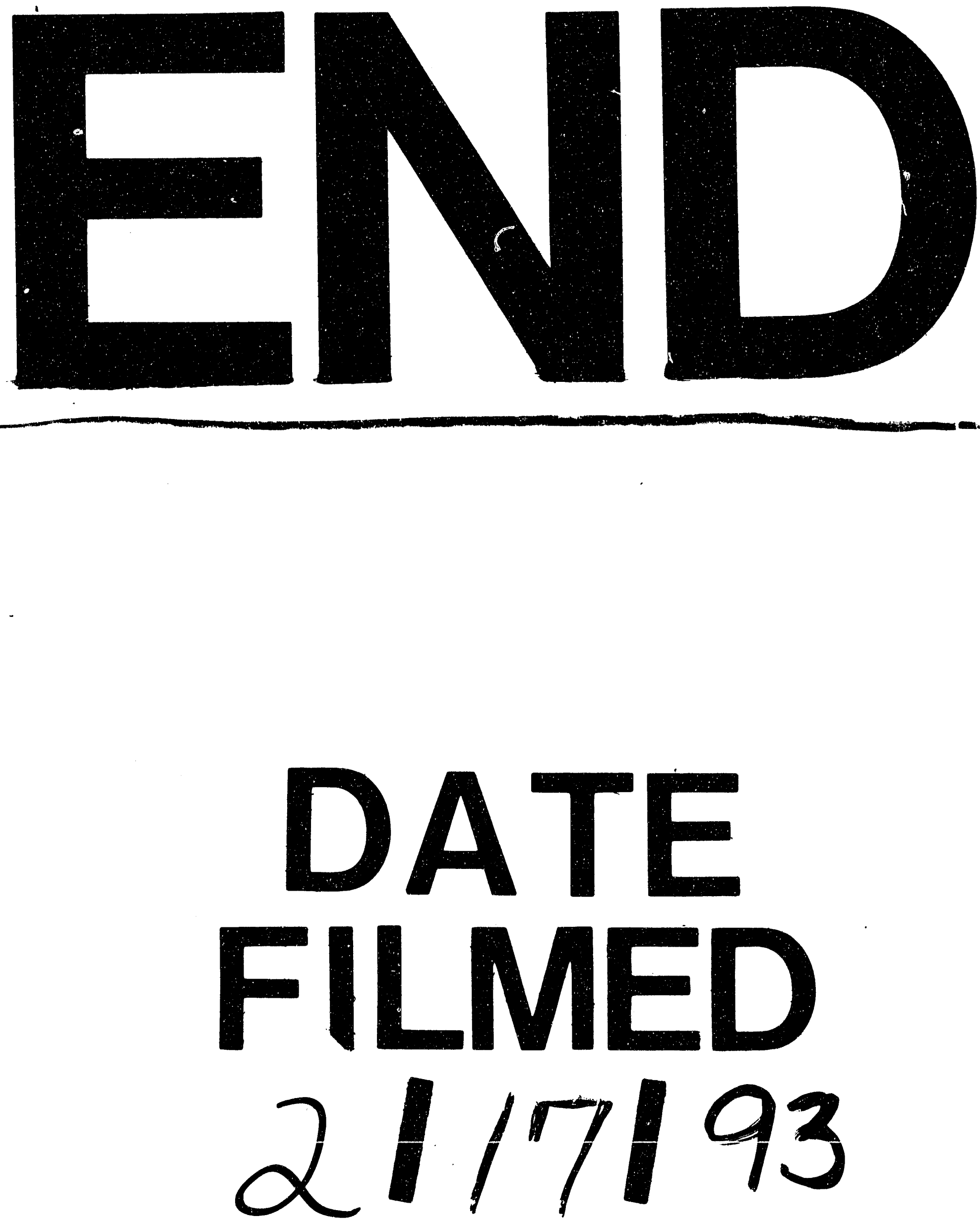
